\title{
Arbeitslosigkeit, riskanter Alkoholkonsum und alkoholbezogene Verhaltensprobleme: eine Längsschnittstudie bei Metallarbeitern
}

\author{
W. Puls \\ M.-L. Inhester \\ J. Reinecke \\ H. Wienold
}

\author{
Unemployment, Risky Alcohol Consumption and Alcohol-Related Behaviour \\ Problems: a Longitudinal Study among Metal Industry Workers
}

\section{Zusammenfassung}

Anliegen: Vor dem Hintergrund der Affekt-Regulations-Hypothese soll die Bedeutung der Arbeitslosigkeit bei der Entwicklung von riskantem Alkoholkonsum abgeklärt werden. Im Rahmen der empirischen Untersuchung wird ein von Peirce, Frone, Russell \& Cooper (1994) entwickeltes Kausalmodell zur Erklärung des Alkoholkonsums und zur Vorhersage alkoholbedingter Probleme getestet. Methode: In einem Längsschnitt in zwei Arbeitsamtsbezirken wurden 1997 zum ersten Messzeitpunkt arbeitslose Metallarbeiter zu psychosozialen Belastungen und zum Konsum von Alkohol befragt, die vorher gegenüber den jeweiligen Arbeitsämtern ihre Bereitschaft zur Teilnahme an der Studie erklärt hatten. 1998 erfolgte die Nachuntersuchung. Für 507 Personen (73,4\% der ersten Messung) liegen vollständige Angaben vor. Ergebnisse: Innerhalb des Untersuchungszeitraums von einem Jahr ergab sich weder ein signifikanter Anstieg des Alkoholkonsums noch eine Zunahme der Alkoholprobleme. Dabei erwies sich die Zugehörigkeit zu einer der Teilgruppen (weiterhin arbeitslos, beschäftigt) als irrelevant. Die Analyse mithilfe linearer Strukturgleichungsmodelle (LISREL) zeigt für die untersuchte Stichprobe, dass sich der Alkoholkonsum zu beiden Messzeitpunkten im Sinne einer Affektregulation erklären lässt, wie dies von der Arbeitsgruppe um Peirce vorgeschlagen worden ist. Schlussfolgerungen: Der Alkoholkonsum ist demnach eine Strategie, um die Depressivität, die zu einem geringen Maße Folge der Arbeitslosigkeit ist, zu bewältigen. Für die Genese der Depressivität ist das Persönlichkeitsmerkmal „Selbstwirk-

\section{Abstract}

Objective: The meaning of unemployment for development of high risk alcohol consumption is to be determined in light of the affect regulation hypothesis. Within the scope of the empirical investigation, a model developed by Peirce, Frone, Russell \& Cooper (1994) explaining alcohol consumption and forecasting problems due to alcohol is tested. Methods: In a longitudinal study in two labour office districts, unemployed metal industry workers, who had previously declared their willingness to the respective labour offices to participate in the study, were initially interviewed in 1997 on their psychosocial stresses and alcohol consumption habits. A follow-up interview was conducted in 1998. Complete information is available for 507 persons ( $73.4 \%$ of the initial interviewees). Results: Within the period of the study of one year, no significant changes in alcohol consumption levels or alcohol problems were found. A change in social status (resumption of employment) was thereby found to be irrelevant. Analysis using linear structural equation models (LISREL) showed for the study sample that alcohol consumption at both interview times can be explained as a form of affect regulation, as has been proposed by the Peirce work group. Conclusions: According to this, alcohol consumption is a strategy for coping with depression which only in small part results from unemployment. For the genesis of this depression, the personality trait of self-efficacy is much more significant than the perceived stresses arising from unemployment. 
samkeit" weitaus bedeutsamer als die perzipierten Belastungen durch die Arbeitslosigkeit.

\section{Schlliusselwörter}

Arbeitslosigkeit · riskanter Alkoholkonsum • Affektregulations-

theorie · Längsschnittstudie · Strukturgleichungsmodell
Key words

Unemployment · risky alcohol consumption · affect regulation theory $\cdot$ longitudinal study $\cdot$ structural equation model

\section{Einleitung}

Angesichts der anhaltenden Massenarbeitslosigkeit und der Verschärfung der sozialen Situation der Arbeitslosen durch die Hartz-IV-Reform gewinnt die Frage nach einem möglichen $\mathrm{Zu}$ sammenhang zwischen Arbeitslosigkeit und Gesundheitsbeeinträchtigungen an Bedeutung. Insbesondere hinsichtlich des Alkoholismus ist dieser Zusammenhang schon seit langem kontrovers diskutiert worden [1]. Die Befundlage ist in hohem Maße durch Widersprüchlichkeiten gekennzeichnet, die eine einheitliche Interpretation der Studien erschweren [2-11]. Einige dieser Studien deuten darauf hin, dass Arbeitslosigkeit nicht oder sogar negativ mit Alkoholgebrauch assoziiert ist [12-21]. Andererseits existiert eine Fülle von empirischen Befunden für eine positive Relation zu Trinkgewohnheiten und Konsumraten [4, 22 -27], zu Verhaltensproblemen als Folge des Alkoholkonsums [4, 26, 28], zu so genannten Alkoholerkrankungen [29, 30] sowie zu Verhaltensstörungen als Folge von Alkoholkonsum [3, 26, 31]. In manchen Studien konnten Zusammenhänge nur für bestimmte Subgruppen aufgewiesen werden, z. B. für allein stehende Personen [32].

Insgesamt scheint es sich bei der Verursachung des Alkoholkonsums durch Arbeitslosigkeit um einen komplexen Prozess zu handeln, der durch zahlreiche Faktoren moderiert wird und sich der Reduktion auf einen einfachen formelhaften Zusammenhang entzieht. Henkel fordert deshalb, „Veränderungen beim Alkoholkonsum nicht wie bisher allein nach quantitativen Merkmalen (z.B. Menge, Frequenz) zu erfassen, sondern auch die Motive, Erwartungen und Intentionen, die dem Alkoholtrinken zugrunde liegen, mit einzubeziehen“ [7].

\section{Theoretischer Bezugsrahmen}

Um die widersprüchlichen Ergebnisse der oben genannten Untersuchungen erklären zu können, erscheint der Bezug auf komplexere Modellvorstellungen notwendig [5, 9, 27, 33]. Ausgangspunkt der folgenden Überlegungen ist in Anlehnung an Peirce et al. die „affect regulation theory“ ([9], vgl. [34]), die man als spezifische Variante stresstheoretischer Erklärungsansätze des Alkoholkonsums interpretieren kann [35]. In dieser theoretischen Perspektive wird Alkohol u.a. zur Reduktion und Bewältigung negativer Emotionen - insbesondere von Depressivität - verwendet. Der Nachweis von Entstehungsbedingungen der Depressivität bildet deshalb ein Kernstück des Modells. Depressivität, die eine Operationalisierung negativer Emotionen darstellt, soll neben persönlichen Ressourcen und sozialer Unterstützung insbesondere durch die finanziellen Belastungen verursacht werden. Negative Gefühle fördern die Bereitschaft, Alkohol zur Stressbewältigung einzusetzen. Die Depressivität selber ist einerseits die Folge ungünstiger Lebensbedingungen (soziale Kon- flikte, fehlende soziale Unterstützung, chronische finanzielle Probleme), andererseits ist jedoch auch eine geringe Selbstwirksamkeit von hoher Bedeutung.

\section{Material und Methoden}

\section{Stichprobe}

In der hier durchgeführten Studie wurde eine Stichprobe älterer arbeitsloser Metallarbeiter untersucht, da hier aufgrund der sozioökonomischen Kontextbedingungen oder der individuellen Bewältigungskompetenzen eine besonders hohe Belastung durch die Arbeitslosigkeit vermutet werden kann. ${ }^{2}$ Riskanter bzw. gefährlicher Konsum wird vor allem von Männern in den Altersgruppen zwischen 40 und 59 betrieben [37]. Durch die Arbeitslosigkeit müsste diese ohnehin schon relativ ungünstige Ausgangslage weiter verschlechtert werden.

Das Anschreiben der Arbeitslosen für die Befragung musste - aus datenschutzrechtlichen Gründen - von zwei Arbeitsämtern vorgenommen werden, in denen ein hoher Anteil arbeitsloser Metallarbeiter registriert war. ${ }^{3}$ Die Bruttostichprobe umfasste männliche, deutsche Arbeitslose in der Altersspanne zwischen 20 bis 60 Jahren und mit einer mindestens sechsmonatigen Dauer der Arbeitslosigkeit. Von den im Jahr 1996 durch die Arbeitsämter angeschriebenen arbeitslosen 3353 Metallarbeitern nahmen fast 20,61\% $(\mathrm{n}=691)$ an der schriftlichen Befragung teil. Die Unterschiede zwischen den Arbeitsamtbezirken waren marginal (Arbeitsamtbezirk West: Bruttostichprobe: 1500; Nettostichprobe: 320; Arbeitsamtbezirk Ost: Bruttostichprobe: 1853; Nettostichprobe: 371). Für eine Befragung von Arbeitslosen kann dies als eine zufriedenstellende Ausschöpfungsquote bewertet werden. Hess et al. konnten bei einem Einsatz von Interviewern mit vergleichbarer Rekrutierung eine Ausschöpfung von 30\% erreichen [38].

Die Nettostichprobe weicht in den Bereichen Familienstand, berufliche Ausbildung und Dauer der Arbeitslosigkeit - wie Über-

\footnotetext{
2 Zum Zeitpunkt der Untersuchung (1997/1998) befand sich die Metallund Elektroindustrie in einer schweren Strukturkrise. Legt man das Jahr 1991 als Maßstab zugrunde, dann hatte Deutschland in sechs Jahren mehr als ein Viertel der Arbeitsplätze in der Metallindustrie verloren [36]. „Hier kumulierten sich die negativen Beschäftigungsfolgen der Krise in Westdeutschland mit dem transformationsbedingten $\mathrm{Zu}$ sammenbruch der ostdeutschen Metallindustrie“ [36].

3 In den Arbeitsamtsbezirken lag die Arbeitslosenquote 1996 bei ca. 10,7\% (Arbeitsamtsbezirk West) bzw. 18,5\% (Arbeitsamtsbezirk Ost). Im Arbeitsamtsbezirk West waren 8,5\% der arbeitslosen Männer Metallarbeiter, im Arbeitsamt Ost 15,4\%. Die Vermittlungsaussichten waren im Arbeitsamt West besonders ungünstig für Arbeitslose mit technischen Berufen, bei denen 1995 zwanzig Arbeitslose pro offener Stelle registriert wurden. Für das Arbeitsamt Ost waren keine entsprechenden Daten zu erhalten.
} 
Tab. 1 Soziodemografische Merkmale: arbeitslose Metallarbeiter der Arbeitsamtsbezirke Ost und West zum Zeitpunkt $\mathrm{t}_{1}$

\begin{tabular}{|c|c|c|c|c|c|c|}
\hline & \multicolumn{2}{|c|}{ Arbeitsamt-Ost } & \multicolumn{2}{|c|}{ Arbeitsamt-West } & \multicolumn{2}{|l|}{ gesamt } \\
\hline & Anzahl & $\%$ & Anzahl & $\%$ & Anzahl & $\%$ \\
\hline \multicolumn{7}{|l|}{ Alter von ... bis } \\
\hline unter 20 & 4 & 1,1 & 3 & 0,9 & 7 & 1,0 \\
\hline $21-24$ & 25 & 6,8 & 12 & 3,8 & 37 & 5,4 \\
\hline $25-29$ & 30 & 8,1 & 31 & 9,7 & 61 & 8,8 \\
\hline $30-39$ & 108 & 29,2 & 63 & 19,7 & 171 & 24,8 \\
\hline $40-49$ & 80 & 21,6 & 57 & 17,8 & 137 & 19,9 \\
\hline $50-60$ & 123 & 33,2 & 154 & 48,2 & 277 & 40,1 \\
\hline \multicolumn{7}{|l|}{$\begin{array}{l}\text { berufliche Ausbildung } \\
\text { (Mehrfachantworten) }\end{array}$} \\
\hline ohne Ausbildung & 12 & 4,5 & 13 & 5,5 & 25 & 5,0 \\
\hline Anlern-Ausbildung & 22 & 7,7 & 23 & 10,6 & 45 & 9,2 \\
\hline betriebliche Ausbildung (Lehre) & 242 & 84,3 & 175 & 80,3 & 417 & 82,6 \\
\hline Fachschule, Fachhochschule & 126 & 20,9 & 108 & 18,3 & 234 & 19,7 \\
\hline \multicolumn{7}{|l|}{ letzte berufliche Stellung } \\
\hline Arbeiter & 91 & 24,7 & 72 & 22,7 & 163 & 23,8 \\
\hline Facharbeiter & 241 & 65,3 & 195 & 61,5 & 436 & 63,6 \\
\hline einfacher Angestellter & 8 & 2,2 & 8 & 2,5 & 16 & 2,3 \\
\hline Angestellter (Sachbearbeiter) & 6 & 1,6 & 9 & 2,8 & 15 & 2,2 \\
\hline Angestellter (hochqualifiziert) & 21 & 5,7 & 30 & 9,5 & 51 & 7,4 \\
\hline Angestellter (leitend) & 2 & 0,5 & 3 & 0,9 & 5 & 0,7 \\
\hline \multicolumn{7}{|l|}{ Dauer der Arbeitslosigkeit } \\
\hline bis 12 Monate & 155 & 43,9 & 80 & 26,5 & 235 & 35,9 \\
\hline 13 bis 24 Monate & 108 & 30,6 & 84 & 27,8 & 192 & 29,3 \\
\hline mehr als 24 Monate & 90 & 25,5 & 138 & 45,7 & 228 & 34,8 \\
\hline
\end{tabular}

prüfungen mithilfe des $\chi^{2}$-Tests zeigten - signifikant von der Bruttostichprobe ab. Es haben vor allem verheiratete, höher qualifizierte und Personen mit längerer Arbeitslosigkeit an der Befragung teilgenommen.

In Tab. 1 ist die realisierte Nettostichprobe hinsichtlich ihrer soziodemografischen Merkmale zum Zeitpunkt $\mathrm{t}_{1}$ dargestellt.

\section{Befragungsinstrumente}

Zum Test des Modells von Peirce et al. wurden Messungen zum Alkoholkonsum, den Problemen mit Alkohol, dem Ausmaß der Trinkmotivation, zur Depressivität, der wahrgenommenen sozialen Unterstützung und sozialen Konflikten, den persönlichen Ressourcen zur Bewältigung von Lebenskrisen und Problemen sowie den finanziellen Belastungen, die sich durch die Arbeitslosigkeit ergeben, durchgeführt [9].

Da die theoretische Grundlage der Konstrukte, die Form der Operationalisierung und die teststatischen Gütekriterien bereits an anderer Stelle ausführlich dargestellt worden sind, soll auf diese Aspekte an dieser Stelle nicht weiter eingegangen werden [39, 40]. Hier muss der Hinweis genügen, dass die Überprüfung der teststatischen Gütekriterien für eine Survey-Studie zufriedenstellende Ergebnisse ergeben hat $[39,40]$.

\section{Auswertungsmethoden}

Es wurden - wie auch von Peirce et al. [9] - die Varianzen und Kovarianzen der Skalen als Datenbasis verwendet und das Mo- dell mit dem LISREL-Ansatz getestet. Die Skalen entsprechen den additiven Indizes der einzelnen Items. Die Kovarianzstrukturanalyse wurde mit der Maximum-Likelihood-(ML-)Schätzung des Programms LISREL 8.0 [41] durchgeführt. Da unterschiedliche Skalierungen der Variablen bzw. Skalen vorliegen, konnte die Methode „Unweighted Least Squares“ nicht verwendet werden [42]. Eine Schätzung der Modelle nach der angemesseneren Methode „Weighted Least Squares“ [43], die die Schiefe der Skalen über den Einbezug der asymptotischen Varianz-KovarianzMatrix hinreichend berücksichtigt, konnte aufgrund der nicht ausreichenden Fallzahlen nicht erfolgen [44]. Eine inhaltliche Interpretation der ML-Parameter ist aufgrund der Stichprobengröße $(n>200)$ trotz vorliegender Verteilungsschiefe der Daten vertretbar [45].

Der LISREL-Ansatz bietet ferner die Möglichkeit, Pfadmodelle gleichzeitig in unterschiedlichen Stichproben zu testen. Jöreskog und Sörbom bezeichnen diese Verwendung des LISREL-Ansatzes als multiplen Gruppenvergleich [41]. Dabei werden zunächst Restriktionen definiert, die gleichzeitig für alle zu analysierenden Gruppen gelten sollten. In einem zweiten Schritt schätzt LISREL dann die Modelle simultan für alle Gruppen unter den zuvor gesetzten Restriktionen. Sämtliche Maße zur Beurteilung des Modellfits beziehen sich auf alle betrachteten Gruppen (hier die weiterhin Arbeitslosen und Personen mit erneuter Beschäftigung) gleichzeitig. Auf diese Weise ist es möglich, die Annahme, dass die ermittelte Kausalstruktur für beide Gruppen gilt, zu testen. 


\section{Deskriptive Befunde}

Bei einem Vergleich der gemessenen Alkoholkonsummengen mit den Ergebnissen der repräsentativen Stichprobe von Herbst et al. zeigt sich, dass die konsumierten Alkoholmengen der $\mathrm{Ar}$ beitslosen in Ost- wie in Westdeutschland erheblich darüber liegen (Tab. 2) [46].

Sowohl die Arbeitslosen als auch die Befragten der repräsentativen Stichprobe nennen in Ostdeutschland höhere konsumierte Alkoholmengen als in Westdeutschland. Bemerkenswerterweise ist auch der Anteil Abstinenter in der Arbeitslosenstichprobe im Vergleich zur repräsentativen Stichprobe der Normalbevölkerung erhöht. Dies bestätigt die Befunde von Lee et al., die eine stärkere Polarisierung des Konsums von Alkohol bei Arbeitslosen im Vergleich zur Normalbevölkerung feststellen konnten [25].

Insgesamt lassen die Befunde den Schluss zu, dass ein deutlich erhöhtes Risiko hinsichtlich der Entwicklung einer Alkoholabhängigkeit bei Teilen der untersuchten Stichprobe arbeitsloser Personen im Vergleich zur Normalbevölkerung besteht. Dieses Resultat stimmt mit zahlreichen anderen Studien überein [6, 33].

\section{und alkoholbedingten Problemen}

Die Längsschnittanalyse basiert auf der Analyse der Kovarianzen des ersten und zweiten Messzeitpunktes unter Einbezug der Kovarianzen zwischen beiden Zeitpunkten. Die Interkorrelationen der unabhängigen Variablen (Variablen 5 bis 8 ) und der abhängigen Variablen (Variablen 1 bis 4) stellt Tab. 3 dar.

Auf der Hauptdiagonalen sind die Korrelationen der Messwiederholung dargestellt. Die relativ geringsten Zusammenhänge bestehen bei der Messung der sozialen Unterstützung und der sozialen Konflikte. Höchste Stabilität besteht bei dem Alkoholkonsum und den Alkoholproblemen.

Die Überprüfung der durch das theoretische Modell nach Peirce et al. [9] vorgegebenen Beziehungen führt sowohl im Quer- wie im Längsschnitt zunächst zu einer wenig zufriedenstellenden Anpassung der erhobenen Daten an das Modell ( $\mathrm{df}=65 ; \chi^{2}=236,46$; $\mathrm{GFI}=0,94 ;$ RMSEA = 0,072; RMR =0,09). Durch Aufnahme zusätzlicher direkter Effekte und Entfernung der Variablen ,finanzielle Belastung“ und „chronische finanzielle Probleme“ (nur beim zweiten Messzeitpunkt) wird eine gute Modellanpassung erreicht $(\mathrm{df}=58$; $\left.\chi^{2}=112,44 ; \mathrm{GFI}=0,92 ; \mathrm{RMSEA}=0,043 ; \mathrm{RMR}=0,04\right)$. Abb. 1 zeigt die Ergebnisse des modifizierten Kausalmodells. Die Abweichungen

Tab. 2 Alkoholmengen der Arbeitslosenstichprobe und einer repräsentativen Stichprobe der Normalbevölkerung im Ost-West-Vergleich [46]

\begin{tabular}{|c|c|c|c|c|c|c|}
\hline \multirow{2}{*}{$\begin{array}{l}\text { tägliche Alkoholmenge in Gramm } \\
\text { Erstbefragung }\left(t_{1}\right) \\
\text { Nachbefragung }\left(t_{2}\right)\end{array}$} & \multicolumn{2}{|c|}{ Arbeitslose Ost } & \multicolumn{2}{|c|}{ Arbeitslose West } & \multicolumn{2}{|c|}{ Vergleich männliche Befragte 1996} \\
\hline & t1 & t2 & t1 & t2 & Ostdeutsche & Westdeutsche \\
\hline kein Konsum & $19,3 \%$ & $17,7 \%$ & $24,9 \%$ & $21,4 \%$ & $12,8 \%$ & $16,3 \%$ \\
\hline 1 - 10 Gramm & $11,0 \%$ & $9,8 \%$ & $10,5 \%$ & $14,6 \%$ & $23,6 \%$ & $31,5 \%$ \\
\hline 11 - 20 Gramm & $14,8 \%$ & $13,6 \%$ & $14,8 \%$ & $14,1 \%$ & $20,1 \%$ & $19,3 \%$ \\
\hline 21 - 40 Gramm & $23,1 \%$ & $25,7 \%$ & $21,5 \%$ & $24,8 \%$ & $23,1 \%$ & $17,8 \%$ \\
\hline 41 - 60 Gramm & $13,3 \%$ & $15,8 \%$ & $8,6 \%$ & $11,6 \%$ & $11,3 \%$ & $7,7 \%$ \\
\hline 61 Gramm und mehr & $18,5 \%$ & $17,4 \%$ & $19,6 \%$ & $13,6 \%$ & $9,2 \%$ & $7,4 \%$ \\
\hline
\end{tabular}

Anmerkung: Signifikanz der Veränderung überprüft durch den t-Test bei gepaarten Stichproben

Bezirk Ost: $\mathrm{t}=-1,73 ; \mathrm{df}=257 ; \mathrm{p}=0,085$ (2-seitig)

Bezirk West: $\mathrm{t}=, 97 ; \mathrm{df}=203 ; \mathrm{p}=0,332$ (2-seitig)

Tab. 3 Wiederholungsbefragung: Korrelationen zwischen den Skalen

\begin{tabular}{|c|c|c|c|c|c|c|c|c|}
\hline $\begin{array}{l}\text { Befragung zu: } \\
\text { Zeitpunkt } t_{2}\end{array}$ & $\begin{array}{l}\text { Zeitpunkt } t_{1} \\
\text { Depressivität }\end{array}$ & Trinkmotive & Alkoholprobleme & Alkoholkonsum & $\begin{array}{l}\text { soz. Unterstüt- } \\
\text { zung }\end{array}$ & $\begin{array}{l}\text { soz. Kon- } \\
\text { flikte }\end{array}$ & $\begin{array}{l}\text { chron. finanz. } \\
\text { Belastung }\end{array}$ & $\begin{array}{l}\text { mangelnde } \\
\text { Ressourcen }\end{array}$ \\
\hline (1) Depressivität & 0,612 & 0,347 & 0,303 & 0,184 & $-0,161$ & 0,353 & 0,294 & 0,496 \\
\hline (2) Trinkmotive & 0,359 & 0,682 & 0,535 & 0,420 & $-0,056$ & 0,266 & 0,196 & 0,393 \\
\hline (3) Alkoholprobleme & 0,249 & 0,480 & 0,753 & 0,431 & $-0,029$ & 0,232 & 0,143 & 0,297 \\
\hline (4) Alkoholkonsum & 0,165 & 0,400 & 0,501 & 0,851 & 0,004 & 0,095 & 0,099 & 0,133 \\
\hline (5) soz. Unterstützung & $-0,133$ & $-0,074$ & $-0,093$ & 0,035 & 0,514 & $-0,103$ & $-0,068$ & $-0,037$ \\
\hline (6) soz. Konflikte & 0,390 & 0,289 & 0,262 & 0,159 & $-0,102$ & 0,571 & 0,272 & 0,462 \\
\hline (7) chron. finanz. Belastung & 0,333 & 0,164 & 0,194 & 0,109 & $-0,137$ & 0,304 & 0,679 & 0,412 \\
\hline (8) mangelnde Ressourcen & 0,483 & 0,395 & 0,336 & 0,165 & $-0,120$ & 0,380 & 0,354 & 0,619 \\
\hline
\end{tabular}

Anmerkung: Alle Korrelationen mit einem Wert über $r=0,15$ sind auf dem Niveau von $p<0,05$ signifikant, alle Korrelationen mit einem Wert über $r=0,18$ sind auf dem Niveau von $\mathrm{p}<0,01$ signifikant. 
vom theoretischen Modell betreffen zusätzliche, auch von Peirce et al. [9] spezifizierte Beziehungen auf die Trinkmotive: Mangelnde persönliche Ressourcen und soziale Konflikte haben hier direkte positive Effekte. Es musste allerdings auch eine weitere Beziehung zwischen mangelnden persönlichen Ressourcen und den Alkoholproblemen aufgenommen werden. Diese drei zusätzlichen Spezifikationen führen zu signifikanten Modellverbesserungen. Die Messwiederholung wurde über direkte Spezifikation der abhängigen Variablen modelliert. Zusätzlich wird eine Modellverbesserung durch einen Cross-Lag-Effekt erreicht: Der Alkoholkonsum des ersten Messzeitpunktes wirkt sich zeitverzögert signifikant auf die Trinkmotive der Wiederholungsbefragung aus. Die Gütekriterien der Modellvarianten belegen deutlich, dass (in beiden Querschnitten) zusätzlich zum hypothetischen Modell direkte Effekte zwischen den unabhängigen Variablen „Ressourcen“ und „soziale Konflikte“ auf die „Trinkmotivation“ sowie zwischen „Ressourcen“ und „Alkoholproblemen“ bestehen.

Anhand der erklärten Varianzen wird deutlich, dass die Stärke des Modells in der Vorhersage von Depressivität (49\% zu t $\mathrm{t}_{1}$ und $46 \%$ $\mathrm{zu} \mathrm{t}_{2}$ ) und Alkoholproblemen ( $44 \% \mathrm{zu} \mathrm{t}_{1}$ und $42 \% \mathrm{zu} \mathrm{t}_{2}$ ) liegt, wobei die Stabilitätsbeziehungen der Variablen keine Berücksichtigung finden. Von der Varianz des Alkoholkonsums können nur 16 bzw. 20\% aufgeklärt werden. Die Varianzaufklärung der Trinkmotive über die direkten Beziehungen von Depressivität, sozialen Konflikten und - am stärksten - durch mangelnde Ressourcen ist im zweiten Messzeitpunkt mit 33\% zufriedenstellend.

Die Frage, ob die Ergebnisse der Längsschnittanalyse bei unterschiedlichen Gruppen bestehen bleiben, wird anhand eines Vergleichs der Gruppe der durchgängig Arbeitslosen und der Personen, die zwischenzeitlich eine Beschäftigung gefunden haben, untersucht. Zwischen diesen beiden Gruppen können strukturelle Unterschiede erwartet werden. Der Einbezug der berenteten Personen in den multiplen Gruppenvergleich verbietet sich darüber hinaus durch die geringe Fallzahl.
Eine Überprüfung der Ergebnisse erfolgt durch den Vergleich der beiden Gruppen mit dem in Abb.1 dargestellten modifizierten Längsschnittmodell. Zusätzlich wurde für die Gruppe der durchgängig Arbeitslosen die Skala „finanzielle Belastung durch Arbeitslosigkeit“ im zweiten Messzeitpunkt hinzugenommen.

Der Effekt der finanziellen Belastung auf die Depressivität für die durchgängig Arbeitslosen beträgt zum zweiten Erhebungszeitpunkt 0,07 (standardisierter Koeffizient). Im Vergleich zum ersten Messzeitpunkt hat sich die Bedeutung dieses Stressors in seiner depressionserzeugenden Wirkung nicht verändert.

Ausgangspunkt der Berechnungen ist die Annahme, dass beide Gruppen identische Kausalstrukturen aufweisen, die durch eine Gleichsetzung aller Parameter für die Kovarianzen der Skalen der durchgängig Arbeitslosen und der Personen, die wieder beschäftigt sind, erfolgte. Variationen zwischen beiden Gruppen wurden systematisch durch getrennte Schätzung 1. der Mittelwertunterschiede der abhängigen Variablen, 2. der Stabilität der abhängigen Variablen und 3. des Cross-Lag-Effektes überprüft.

Eine (geringe) Modellverbesserung anhand unterschiedlicher Mittelwerte in den abhängigen Variablen besteht allein für die Messung des Alkoholkonsums im ersten Messzeitpunkt. Darüber hinaus führte die Annahme gleicher Mittelwerte der abhängigen Variablen zu vergleichbaren Modellanpassungen wie unter der Annahme, dass die Mittelwerte variieren.

Die Stabilitätsbeziehungen der abhängigen Variablen differieren kaum zwischen beiden Gruppen. Selbst für die Beziehung zwischen der Depressivität der ersten und zweiten Erhebung, die bei den durchgängig Arbeitslosen über den Erhebungszeitraum unverändert und bei den wieder Beschäftigten gesunken ist, wird keine Modellverbesserung durch getrennte Schätzung erreicht. Die unstandardisierten Pfadkoeffizienten betragen bei getrennter Schätzung 0,36 für die Gruppe der durchgängig Arbeitslosen und

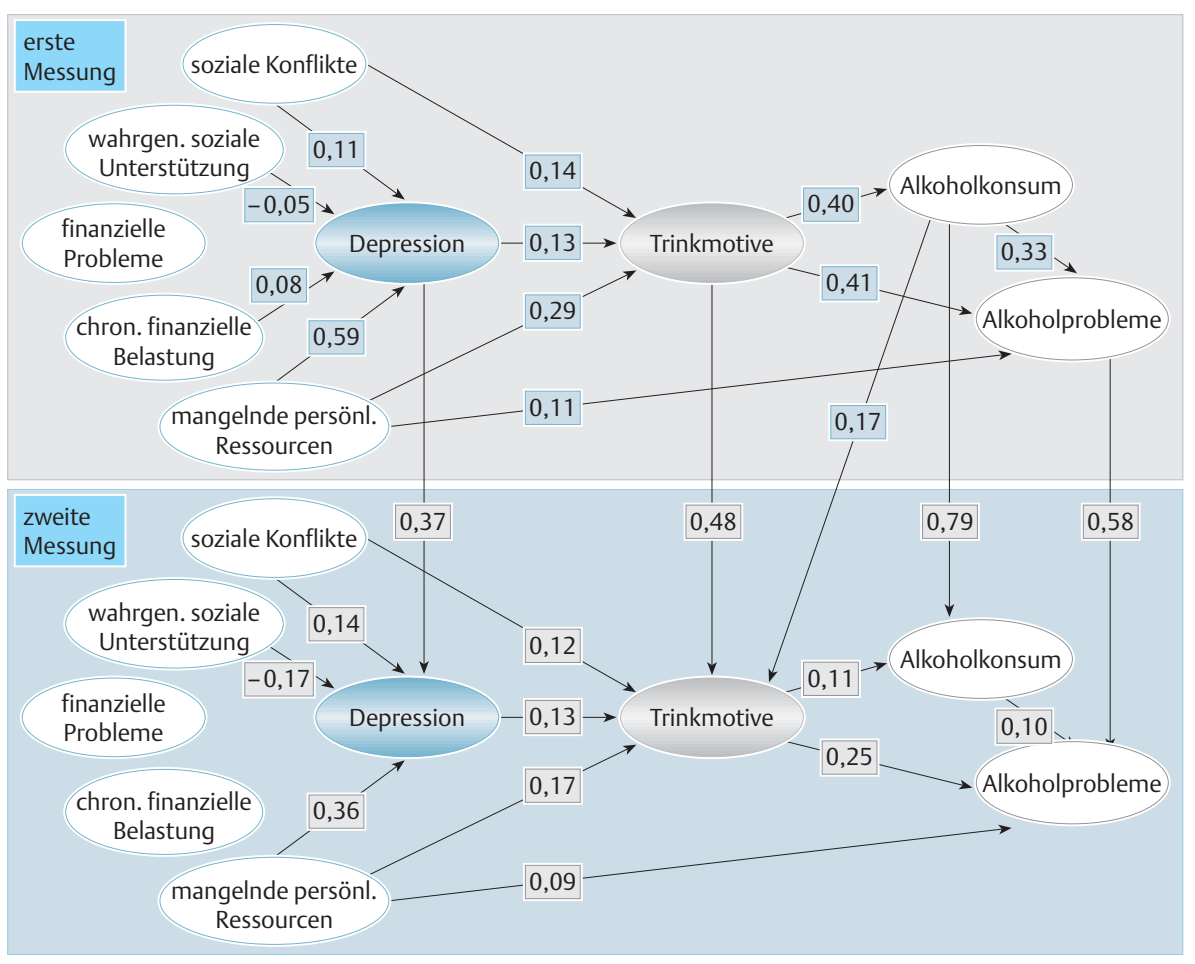

Abb. 1 Längsschnittmodell zum Konsum von Alkohol bei Arbeitslosigkeit. 
0,34 für die wieder Beschäftigten. Die größte Abweichung findet sich in der Stabilitätsbeziehung der Trinkmotive: Bei durchgängig Arbeitslosen fällt die Stabilität der Trinkmotive mit 0,48 geringer aus als bei Personen, die zwischenzeitlich beschäftigt sind. Hier beträgt die Stabilität 0,54 (unstandardisierter Pfadkoeffizient).

Die getrennte Schätzung des Cross-Lag-Effekts weist ebenfalls darauf hin, dass die vorgegebene Modellstruktur bei beiden Gruppen identisch ist. Die Auswirkung des Alkoholkonsums auf die ein Jahr später erhobene Trinkmotivation beträgt bei den durchgängig Arbeitslosen 0,008, bei den wieder Beschäftigten 0,011 (unstandardisierte Koeffizienten).

Der multiple Gruppenvergleich bestätigt die Gültigkeit des Modells für die Gruppe der durchgängig Arbeitslosen und der Personen, die wieder beschäftigt sind.

\section{Diskussion}

Das zentrale Ergebnis der Studie besteht 1. darin, dass sich das Modell sowohl auf die Gruppe der Arbeitslosen wie auch der Beschäftigten anwenden lässt, und 2. ist hervorzuheben, dass ein hoher Alkoholkonsum - mit zeitverzögerter Wirkung - zu einer stärkeren Trinkmotivation (siehe Abb.1) führt. Ferner konnte 3. kein Effekt der Depressivität zu $\mathrm{t}_{1}$ auf den Alkoholkonsum zu $\mathrm{t}_{2}$ nachgewiesen werden, sondern vielmehr bewirkt der Konsum $\mathrm{zu} \mathrm{t}_{1}$ eine Stabilisierung der Motive $\mathrm{zu}_{2}$

Die Ergebnisse stellen deshalb die vermutete starke Abhängigkeit negativer Emotionen von finanziellen Einschränkungen und sozialen Unterstützungen im Zuge der Arbeitslosigkeit infrage. Dieses Resultat findet sich ebenfalls in der Untersuchung von Peirce et al. [9]. Dies kann man so interpretieren, dass im Rahmen des hier untersuchten Zeitfensters weniger die nachteiligen Umstände selbst (hier bedingt durch die Arbeitslosigkeit), sondern die individuelle Bewältigungskompetenz von Problemen einen wesentlicheren Einfluss auf den Konsum von Alkohol hat.

Depressive Verstimmungen verfestigen offenbar nur in begrenztem Umfang die Motive, Alkohol zum Coping zu verwenden. Der Zusammenhang zwischen Arbeitslosigkeit und Alkoholkonsum ist vermutlich weniger Folge spezifischer Belastungen im Zuge der Arbeitslosigkeit, sondern wahrscheinlich war die Prävalenz vor dem Eintritt der Arbeitslosigkeit gegenüber dem Bundesdurchschnitt erhöht. Welche Gründe können hierfür maßgeblich sein?

Man kann vermuten, dass ein Teil der Befragten bereits im Zuge der Entlassung seinen Konsum gesteigert hat. Hierfür kann zunächst angeführt werden, dass Arbeitsplatzunsicherheit starke Effekte auf die psychische Befindlichkeit hat [47] und möglicherweise indirekt auch den Alkoholkonsum beeinflusst. Diese Sichtweise wird durch den Befund gestützt, dass berufliche Gratifikationskrisen, die z.B. durch drohenden Arbeitsplatzverlust ausgelöst werden können, das Risiko für Alkoholmissbrauch drastisch erhöhen $[48,49]$. Auch in anderen Studien konnte gezeigt werden, dass der Verlust des Arbeitsplatzes stärker als anhaltende Arbeitslosigkeit zum missbräuchlichen Konsum von Alkohol disponiert [50]. Dooley, Catalano und Hough kamen bei der Analyse des „Epidemiologic Catchment Area (ECA) survey“ $(n=10534)$ zu dem Ergebnis, dass insbesondere der Verlust der Arbeit einen sehr deutlichen Anstieg des Risikos von alkoholbedingten Störungen bewirkt [31].

Die hier skizzierten Befunde stützen die Annahme, dass der Konsum bereits im Vorfeld der drohenden Arbeitslosigkeit ansteigt und dies partiell zu einem Deckeneffekt führt, d. h. durch die Chronifizierung der Belastungen im Zuge der Arbeitslosigkeit tritt kein bedeutsamer weiterer Anstieg des Konsums ein. Für eine Gesundheitsförderung, die die Belastungen durch Arbeitslosigkeit mindern will, bedeutet dies, dass die Interventionen - um wirksam zu sein - früher beginnen müssen, d. h. in diesem Fall, dass die Stabilisierung der Selbstwirksamkeit und des kontrollierten Konsums von psychoaktiven Substanzen bereits erfolgt sein muss, bevor diese spezifischen Belastungen durch drohenden Arbeitsplatzverlust oder durch die Arbeitslosigkeit einsetzen [51].

Parallel zur Untersuchung der arbeitslosen Metallarbeiter wurde deshalb eine Studie in Betrieben der metallverarbeitenden Industrie durchgeführt, in denen betriebsbedingte Kündigungen drohten [48]. Gegenstand war die Untersuchung von drohendem Arbeitsplatzverlust bzw. Arbeitslosigkeit, die damit verbundenen Stresswahrnehmungen und die Konsequenzen dieser Wahrnehmungen auf den Konsum von Alkohol, Zigaretten und psychoaktiven Medikamenten. Es konnte gezeigt werden, dass die Theorie der Gratifikationskrise von Siegrist [52], die ursprünglich und mit Erfolg zur Erklärung von kardiovaskulären Erkrankungen entwickelt worden war, auch geeignet ist, den stressbedingten Konsum von Alkohol partiell aufzuklären, der im Zuge betrieblicher Umbruchprozesse zu erwarten ist [10].

In einer Interventionsstudie - die zum Teil in Werken durchgeführt wurde, in denen betriebsbedingte Kündigungen drohten - wurde die suchtpräventive Wirkung eines spezifischen Stressbewältigungsprogramms überprüft, das von der Theorie der Gratifikationskrise abgleitet worden ist [53] und um Komponenten aus dem Programm von Schelp, Gravemeier und Maluck [54] ergänzt wurde. Das Programm zielte insbesondere a) auf den Abbau übersteigerter Kontrollambitionen, b) die Veränderung der Wirkungserwartungen an den Alkoholkonsum (Trinkfunktionen) und c) den Alkoholkonsum selbst [55]. Die Überprüfung des Programms erfolgte auf der Grundlage einer längsschnittlichen Interventionsstudie mit drei Messzeitpunkten, an der 105 Beschäftigte teilnahmen, die zu Beginn der Studie randomisiert wurden. Im Rahmen der Follow-up-Untersuchung zeigte sich nach drei Monaten eine signifikante Reduzierung des Alkoholkonsums, der Kontrollambitionen und spezifischer Wirkungserwartungen an den Alkoholkonsum [56, 57].

Daraus kann abschließend die Schlussfolgerung gezogen werden, dass die Interventionen vor dem Eintritt der Arbeitslosigkeit einsetzen sollten, möglicherweise als Angebote im Rahmen von Outplacement/Replacement-Konzepten, die u.a. den psychosozialen Stress in der beginnenden Arbeitslosigkeit abzumildern versuchen [58]. Haben sich die Bewältigungsstrategien (z. B. riskanter Alkoholkonsum) wie in der hier untersuchten Stichprobe habitualisiert, dann sind Veränderungen - wenn überhaupt nur noch durch sehr aufwändige sozialpolitische und personenbezogene Interventionen erreichbar [8]. 


\section{Literatur}

${ }^{1}$ Henkel D. Die Trunksucht ist die Mutter der Armut - zum immer wieder fehlgedeuteten Zusammenhang von Alkohol und Armut in Deutschland vom Beginn des 19. Jahrhunderts bis zur Gegenwart. In: Henkel D (Hrsg). Sucht und Armut. Alkohol, Tabak, illegale Drogen. Opladen: Westdeutscher Verlag, 1998: 13-79

${ }^{2}$ Catalano RA. Gesundheitseffekte wirtschaftlicher Unsicherheit: Ein analytischer Überblick. In: Kieselbach T, Voigt P (Hrsg). Systemumbruch, Arbeitslosigkeit und individuelle Bewältigung in der ExDDR. Weinheim: Deutscher Studien Verlag, 1993: 84-94

${ }^{3}$ Catalano R, Dooley D, Wilson G et al. Job loss and alcohol abuse: a test using data from the epidemiologic catchment area project. Journal of Health and Social Behaviour 1993; 34: 215 - 225

${ }^{4}$ Crawford A, Plant MA, Kreitman N et al. Unemployment and drinking behaviour: some data from a general population survey of alcohol use. Br J Addict 1987; 82: 1007-1016

${ }^{5}$ Forcier MW. Unemployment and alcohol abuse: a review. J Occup Med 1988; 30: 246-251

${ }^{6}$ Henkel D. Arbeitslosigkeit, Alkoholkonsum und Alkoholabhängigkeit: Nationale und internationale Forschungsergebnisse. In: Henkel D, Vogt I (Hrsg). Sucht und Armut. Alkohol, Tabak, Medikamente, illegale Drogen. Opladen: Leske und Budrich, 1998: 101-136

${ }^{7}$ Henkel D. Arbeitslosigkeit, Alkoholkonsum und Alkoholabhängigkeit. Forschungsergebnisse, Defizite, Hypothesen. Abhängigkeiten 1998; 3 : 9-29

${ }^{8}$ Henkel D. Alkohol- und Tabakprävention für Arbeitslose, Arme und Obdachlose. In: Schmidt B, Hurrelmann K (Hrsg). Präventive Suchtund Drogenpolitik. Opladen: Westdeutscher Verlag, 2000: 163-192

${ }^{9}$ Peirce R, Frone M, Russell M et al. Relationship of financial strain and psychosocial resources to alcohol use and abuse: The mediating role of negative affect and drinking motives. Journal of Health and Social Behaviour 1994; 35: 291 - 308

10 Puls W. Massenarbeitslosigkeit, Stress und der riskante Konsum von Alkohol. Versuch einer soziologischen Erklärung auf der Grundlage des Gratifikationskrisenkonzeptes. Journal of Public Health 2004; 12 (4): 259-270 (DOI: 10.1007/s10389-004-0029-2)

11 Winton M, Heather N. Effects of unemployment on drinking behavior: a review of the relevant evidence. Int J Addict 1986; 12: 1261 - 1283

${ }^{12}$ Cooke DG, Bartley MJ, Cummins RO et al. Health of unemployed middle-aged men in Great Britain. Lancet 1982; 1 (8284): 1290-1294

${ }^{13}$ Cooke DG, Allan CA. Self-Reported alcohol consumption and dissimilation in a Scottish urban sample. J Stud Alcohol 1983; 44 (4): 617-629

${ }^{14}$ Hammer T. Unemployment and use of drug and alcohol among young people: a longitudinal study in the general population. $\mathrm{Br}$ J Addict 1992; 87: $1571-1581$

${ }^{15}$ Heather N, Laybourn P, MacPherson B. A prospective study of the effects of unemployment on drinking behaviour. Soc Psychiatry 1987; 22: $226-233$

${ }^{16}$ Iversen L, Klausen $\mathrm{H}$. Alcohol consumption among laid-off workers before and after closure of a danish ship-yard: a 2-year follow-up study. Soc Sci Med 1986; 22 (1): 107-109

17 Lester D. Unemployment and alcohol consumption. Psychol Rep 1996; 79: 150

${ }^{18}$ Morris JK, Cook DG, Shaper AG. Non-employment and changes in smoking, drinking, and body weight. BMJ 1992; 304: 536-541

19 Plant MA. Drinking careers. Occupations, drinking habits, and drinking problems. London: Tavistock Publications, 1979

${ }^{20}$ Rehm J, Gmel G. Patterns of alcohol consumption and social consequences. Results from an 8-year follow-up study in Switzerland. Addiction 1999; 94 (6): 899-912

${ }^{21}$ Warr P, Payne R. Social class and reported changes in behavior after job loss. J Appl Soc Psychol 1983; 13: 206 - 222

22 Bloomfield K. West german drinking patterns in 1984 and 1990. Eur Addict Res 1998; 4: 163-171

${ }^{23}$ Bray JW, Zarkin GA, Dennis ML et al. Symptoms of dependence, multiple substance use, and labor market outcomes. Am J Drug Alcohol Abuse 2000; 26: 77-95

${ }^{24}$ Henkel D. Zum Konsum von Alkohol, Tabak und psychoaktiven Medikamenten und Einkommensarmen: eine Auswertung des Nationalen Gesundheitssurveys 1991/1992 der Bundesrepublik Deutschland. Abhängigkeiten 2000; 1: 26-43
${ }^{25}$ Lee AJ, Crombie IK, Smith WCS et al. Alcohol consumption and unemployment among men: the Scottish heart health study. Br J Addict 1990; 85: $1165-1170$

${ }^{26}$ Montgomery SM, Cook DG, Bartley MJ et al. Unemployment, cigarette smoking, alcohol consumption and body weight in young British men. Eur J Public Health 1998; 8: 21 - 27

27 Power C, Estaugh V. Employment and drinking in early adulthood: a longitudinal perspective. Br J Addict 1990; 85: 487-494

${ }^{28}$ Smart RG. Drinking problems among employed, unemployed and shift workers. J Occup Med 1979; 21: 731 - 736

${ }^{29}$ Brenner MH. Economic change, alcohol consumption and heart disease mortality in nine industrialized countries. Soc Sci Med 1987; 25 (2): $119-132$

${ }^{30}$ Heien D, Pompelli G. Stress, ethnic and distribution factors in a dichotomous response model of alcohol abuse. J Stud Alcohol 1987; 48: $450-455$

31 Dooley D, Catalano R, Hough R. Unemployment and alcohol disorder in 1910 and 1990: drift versus social causation. Journal of Occupational and Organizational Psychology 1992; 65: 277-290

${ }^{32}$ Luoto R, Poikolainen K, Uutela A et al. Unemployment, sociodemographic background and consumption of alcohol before and during the economic recession of the $1990 \mathrm{~s}$ in Finnland. Int J Epidemiol 1998; 27: $623-629$

${ }^{33}$ Henkel D. Arbeitslosigkeit und Alkoholismus. Epidemiologische, ätiologische und diagnostische Zusammenhänge (Psychologie sozialer Ungleichheit, Bd 3). Weinheim: Deutscher Studien Verlag, 1992

${ }^{34}$ Cappell H, Greeley J. Alcohol and tension reduction: an update on research and theory. In: Blane HAT, Leonard KE (Hrsg). Psychological theories of drinking and alcoholism. New York: The Guilford Press, 1987: $15-54$

35 Puls W. Arbeitsbedingungen, Stress und der Konsum von Alkohol. Opladen: Leske + Budrich, 2003

${ }^{36}$ Industriegewerkschaft Metall (Hrsg). Daten für Metaller 1998. Frankfurt/Main, 1998

37 Bühringer G, Augustin R, Bergmann E et al. Alkoholkonsum und alkoholbezogene Störungen in Deutschland. Baden-Baden: Nomos, 2000

${ }^{38}$ Hess D, Hartenstein W, Smid M. Auswirkungen von Arbeitslosigkeit auf die Familie. Mitt Arbeitsmarkt Berufsforsch 1991; 1: 178-193

39 Puls W, Inhester ML, Reinecke J et al. Alkoholkonsum und alkoholbezogene Verhaltensprobleme bei arbeitslosen Metallarbeitern in der Perspektive der Affekt-Regulations-Hypothese. Sucht 1999; 45: $390-405$

${ }^{40}$ Puls W, Ulbrich T, Wienold H. Skalen zur Arbeit, Arbeitslosigkeit und Gesundheit. [Unveröffentlicht] Nr. 2, Aktuelle Beiträge zur Soziologie, Institut für Soziologie, Westfälische Wilhelms-Universität, 2000. Im PDF-Format zugänglich unter „http://edok01.tib.uni-hannover.de/ edoks/e01fb02/346 456 185.pdf"

${ }^{41}$ Jöreskog KG, Sörbom D. LISREL 8: Structural equation modeling with the Simplis command language. Chicago: Scientific Software International, 1993

${ }^{42}$ Bollen KA. Structural equations with latent variables. New York: Wiley, 1989

43 Browne MW. Asymptotically distribution-free methods for the analysis of covariance structures. Br J Math Stat Psychol 1984; 37: 62 - 83

${ }^{44}$ Urban D, Mayerl J. Wie viele Fälle werden gebraucht? Ein Monte-Carlo-Verfahren zur Bestimmung ausreichender Stichprobengrößen und Teststärken (power) bei Strukturgleichungsanalysen mit kategorialen Variablen. ZA-Information 2003; 53: 42 - 69

45 Boomsma A. On the robustness of LISREL (maximum likelihood estimation) against small sample size and non-normality. [Unveröffentlichte Dissertation]. Niederlande: Universität Groningen, 1983

${ }^{46}$ Herbst K, Kraus L, Scherer K. Repräsentativerhebung zum Gebrauch psychoaktiver Substanzen bei Erwachsenen in Deutschland. Schriftliche Erhebung 1995. München: Institut für Therapieforschung, 1996

${ }^{47}$ Mohr G. Erwerbslosigkeit, Arbeitsplatzunsicherheit und psychische Befindlichkeit. Frankfurt: Peter Lang, 1997

48 Puls W, Wienold H, Blank T. Die Einwirkung von Gratifikationskrisen am Arbeitsplatz auf den Konsum von Alkohol: Eine schriftliche Befragung in Betrieben der metallverarbeitenden Industrie. Sucht 1998; 44: $183-199$

${ }^{49}$ Stansfeld S, Head J, Marmot M. Work-related factors and ill health. The Whitehall II Study. [Unveröffentlichter Bericht]. London: University College, 2000

${ }^{50}$ Dooley D, Prause J. Underemployment and alcohol misuse in the national longitudinal survey of youth. J Stud Alcohol 1998; 59: 669-680 
${ }^{51}$ Schwarzer R. Psychologie des Gesundheitsverhaltens. Göttingen: Hogrefe, 1996

52 Siegrist J. Soziale Krisen und Gesundheit: eine Theorie der Gesundheitsförderung am Beispiel von Herz-Kreislauf-Risiken im Erwerbsleben (Gesundheitspsychologie, Bd 5). Göttingen: Hogrefe, 1996

53 Siegrist K, Silberhorn T. Streßabbau in Organisationen - ein Manual zum Streßmanagement. Münster: Lit-Verlag, 1998

${ }^{54}$ Schelp T, Gravemeier R, Maluck D. Rational-Emotive Therapie als Gruppentraining gegen Stress: Seminarkonzepte und Materialien. Bern: Huber, 1997

${ }^{55}$ Wiegard U, Tauscher N, Inhester ML et al. Gelassen bei der Arbeit. Ein Trainingskurs zur Bewältigung von Stress am Arbeitsplatz. [Unveröffentlicht] Nr. 1, Aktuelle Beiträge zur Soziologie, Institut für Soziologie, Westfälische Wilhelms-Universität 2000. Im PDF-Format zugänglich unter „http://edok01.tib.uni-hannover.de/edoks/e01fb02/ 346 519 276.pdf“"
${ }^{56}$ Puls W, Inhester ML, Wienold H. Entwicklung und Testung eines Stressbewältigungstrainings zur Reduktion des Alkoholkonsums. In: Deutsche Hauptstelle gegen die Suchtgefahren (Hrsg). Sucht und Arbeit - Prävention und Therapie substanzbezogener Störungen in der Arbeitswelt. Freiburg: Lambertus-Verlag, 2001: $73-88$

${ }^{57}$ Puls W, Inhester ML et al. Stressbewältigungstrainings als Komponente betrieblicher Suchtprävention. Sucht 2002; 4: 271 -283

${ }^{58}$ Kieselbach T. Sozialer Konvoi und nachhaltige Beschäftigungsfähigkeit: Perspektiven eines zukünftigen Umgangs mit beruflichen Transitionen. In: Zempel J, Bacher J, Moser K (Hrsg). Erwerbslosigkeit. Ursachen, Auswirkungen und Interventionen. Opladen: Westdeutscher Verlag, 2001: $382-396$ 